\title{
La ciudad contemporánea: problemas y tendencias. Perspectivas institucionales, sociales y artísticas
}

\author{
The contemporary city: problems and trends. \\ Institutional, social and artistic perspectives \\ LAURA LUQUE RODRIGO \\ Universidad de Jaén \\ ORCID: https://orcid.org/0000-0003-2651-6948 \\ lluque@ujaen.es
}

\section{RESUMEN}

Este texto aborda la ciudad contemporánea desde distintas perspectivas para tratar algunos de sus problemas actuales: la pérdida de espacio público, la preeminencia del tráfico rodado en las vías de circulación, la gentrificación en los centros históricos y la falta de seguridad para las mujeres. Para ello, se tratan las posturas desde las instituciones, así como algunas soluciones propuestas por la propia ciudadanía y sobre todo desde el mundo del arte. Por último, se aborda el tema de los grandes proyectos arquitectónicos que buscan la espectacularidad, ligados en muchos casos al arte contemporáneo.

Palabras clave: Espacio público, urbanismo, arte urbano, museos contemporáneos, género.

\begin{abstract}
This text approaches the contemporary city from different perspectives to treat some of his current problems: the loss of public space, the preeminence of the traffic rolled in the routes of traffic, the gentrification in the historical centers and the safety lack for the women. For it, the positions treat each other from the institutions, as well as some solutions proposed by the own citizenship and especially from the world of the art. Finally, it deals with the topic of great architectural projects that seek spectacularity, linked in many cases to contemporary art.
\end{abstract}

Keywords: Public space, urbanism, urban art, contemporary museums, genre. 


\title{
1. INTRODUCCIÓN
}

\author{
«Las personas construyen edificios y ciudades, \\ y de la misma manera estos construyen a las personas». \\ (ARTEAGOITIA, 2014, pp.186-187)
}

\subsection{Cuestiones terminológicas e históricas sobre la ciudad y el espacio urbano}

En el título de este trabajo se emplea el término «ciudad contemporánea», expresión contrapuesta a la de «ciudad histórica». Esta última locución, muy usada en los textos científicos e incluso legislativos - como la Carta de Cracovia (2000), por ejemplo-, no tiene sin embargo una definición clara y precisa. ¿A qué nos referimos los historiadores del arte, arquitectos o urbanistas cuando hablamos de la ciudad histórica? ¿Y de la ciudad contemporánea? ¿Qué características tiene que cumplir una población para poder referirse a ella bajo estas nomenclaturas? Monterroso define la ciudad histórica como «el resultado de la acción humana en un entorno construido - un paisaje artificial y humanizado- determinada por las circunstancias sociales, económicas, históricas y culturales por las que ha tenido que pasar» (MONTERROSO, 2011, p. 43). Según este axioma, podríamos llamar ciudad histórica a cualquier población. Otros autores hablan de ciudades patrimoniales, término que aparece también empleado por instituciones internacionales del patrimonio, como la UNESCO; con esta otra locución parecen reducir más el significado, para centrarse en aquellas poblaciones con centros históricos con una entidad considerada importante, pero que no entran en el grupo reconocido como Ciudades Patrimonio de la Humanidad.

Por otro lado, tendríamos que partir de la propia definición de ciudad, tarea ardua dado que, aun siendo un tema tratado durante largo tiempo, no se ha conseguido consenso entre investigadores o teóricos. Una buena forma de conceptualizar la ciudad es como «una comunidad de asentamiento base sedentario que se caracteriza por la ubicación de lugares de encuentro, de espacios sociales singulares de carácter político y/o ideológico, donde se realizan prácticas sociales que involucran a otras comunidades, fundamentalmente a las comunidades no urbanas de su territorio» (CASTRO, ESCORIZA, OLTRA, OTERO, SANAHUJA, 2003, p. 7). En cualquier caso, parece imprescindible cuando hablamos de ciudad, contar con un equipamiento básico que incluya el ocio y el transporte; en orden de subsistencia, sus habitantes dependen de producciones alimentarias que vienen de fuera; deben existir espacios de encuentro vinculados a diversas actividades así como espacios de circulación; y por supuesto requiere la presencia de un órgano de poder, de hecho, durante la Edad Media se llamaron civitas, a aquellas localidades en las que residía el obispo (MARTÍNEZ TABOADA, 1985, p. 959).

El urbanismo es la forma en que la ciudad se expresa, y en él encontramos dos tipos de espacios, aquellos que adquieren su cualificación por la identidad que le proporcionan los propios actores y los espacios de representación, aquellos vinculados al poder, mucho más homogéneos en su contenido (GONZÁLEZ DE CASTELLS, 2013). Para Fernández, 
«el espacio público es aquel que el habitante de la ciudad -habitual o esporádico- puede utilizar sin más limitaciones que ciertas ordenanzas legales y ateniéndose a unas normas de decoro aceptadas por el conjunto social» (FERNÁNDEZ, 1988, p. 35), si bien esta definición puede resultar simplista, nos da algunas pistas sobre el uso del espacio público.

En el siglo XX, el filósofo Gaston Bachelard planteó que el espacio existe por medio del sujeto, que es algo más que un simple ser contemplativo (BACHELARD, 2000). Estas teorías influyeron notablemente en Foucault, quien creó el ineludible término de heterotopía, que define la ciudad contemporánea como un conjunto de espacios heterogéneos de lugares y relaciones (FOUCAULT, 1967). Lefebvre definió el espacio en la ciudad capitalista, como fragmentario y jerarquizado y tendente a la homogeneización, si bien aclara que ningún espacio es igual a otro. Asimismo, relacionando espacio y tiempo, se ocupa de la 'complejificación' de la sociedad en su paso de rural a industrial y de industrial a urbana, que al deber encajar en un espacio abstracto que tiende a lo homogéneo, entra en conflicto (LEFEBVRE, 1972).

Pero las sociedades que habitan las ciudades son cada vez más heterogéneas, por lo que según apunta Aguilar Rocha, «los espacios generados desde este pensamiento moderno y que dio lugar al capitalismo no permite la vinculación con los seres humanos y el mundo. Esto tiene como consecuencias la psicosis en los individuos y en las sociedades. Los espacios así entornados dejan de ser habitables» (AGUILAR ROCHA, 2017, p.100). Desde este punto, el autor aborda el concepto de habitar. Partiendo de Heidegger, tiene que ver con el vínculo del individuo con el mundo, con un lugar concreto (HEIDEGGER, 1994, p.127). Pero si hablamos de la ciudad capitalista, también debemos atender a los postulados marxistas, que no se ocupan sino de proporcionar a los ciudadanos vivienda y servicios. Fue Engels quien más se interesó por este tema, sin embargo, no se plantea una planificación urbana como tal.

Pero ¿qué sucede con los espacios en la ciudad contemporánea? Para González Martínez, «las nuevas infraestructuras, servicios y equipamientos, los nuevos modos de ocio, consumo y vivienda, han de incorporarse a la ciudad histórica. Han de hacerlo de manera consciente, tan reivindicativa de su condición actual, como atenta a su emplazamiento, buscando cultivar una vecindad que nuestra cultura democrática reconoce anclada no en la homogeneidad, sino en el respeto profundo a las diferencias» (GONZÁLEZ MARTÍNEZ, 2015, p. 202). Es por tanto esa integración de la diversidad social, así como de la nueva edilicia en la ciudad histórica, uno de los retos de la ciudad contemporánea.

El mayo del '68 supuso una renovación en la forma de entender la ciudad, frente a la importancia del tiempo y la preservación del patrimonio desarrollada tras las Guerras Mundiales (reflejadas en las cartas internacionales). El situacionismo da importancia al espacio. Los planteamientos de Debord desde la psicogeografía, llevan al ciudadano a huir de la rutina y experimentar la ciudad a través de las emociones (DEBORD, 1958), a través de las derivas, que difieren del deambular surrealista, pues reflejan una «situación urbana objetiva de interés o aburrimiento» (PERNIOLA, 2008, 25). La Internacional Situacionista propone una renovación urbana que se opone al funcionalismo, pues conside- 
ran que los arquitectos funcionalistas construyen ciudades que condenan a la población al aburrimiento o al aislamiento.

En este sentido, Constant plantea «New Babylon», una ciudad utópica construida para una nueva especie, el Homo Ludens, un ser creativo liberado del trabajo cotidiano y donde la arquitectura tradicional y las instituciones se habrían desintegrado, precisamente en 1956, cuando las ciudades europeas se estaban reconstruyendo tras la II Guerra Mundial. La idea de crear una ciudad utópica viene desde antiguo, muchas son las aportaciones durante la Edad Moderna, no hay más que mencionar la Sforzinda de Filarete o a nivel político las utopías platónicas, agustinianas, la de Tomás Moro o Sinapia ${ }^{1}$. Sin embargo, todas ellas están marcadas por el deseo de ordenar a la sociedad de una manera práctica, regular, a diferencia de New Babilon que es incluso una ciudad nómada, donde sus habitantes podrían recrear la ciudad a su gusto de una manera espontánea (WEB MACBA). Esta ciudad comparte algunas cuestiones con los planteamientos de Le Corbusier, como estar elevada, permaneciendo la circulación por debajo de los espacios en los que transcurre la vida.

Los historiadores del arte nos hemos preocupado históricamente por la morfología de la ciudad como proponía Aldo Rossi y autores españoles como Bonet Correa; nos hemos esforzado por describir su equipamiento, sus construcciones y espacios, pero en la ciudad contemporánea se abren otros campos, como el propio deambular o el devenir, que sí ha sido objeto de reflexión por filósofos y artistas en el siglo XX.

\subsection{Ciudades históricas y sus relaciones institucionales y sociales. Identidad y protección del patrimonio}

«La época actual se enfoca en el espacio, sobre todo en lo histórico, en el tiempo». (NAVARRO FUENTES, 2016, p. 101)

Monterroso señala como es la dimensión humana la que da sentido a la ciudad histórica (MONTERROSO, 2011, p. 41). Los centros históricos y sus monumentos muestran una memoria colectiva que proporciona identidad a la sociedad que los habita, fomenta la cohesión y la identificación entre miembros de una misma comunidad, junto con otros elementos propios del patrimonio inmaterial. Es por ello que tanto a nivel internacional como más local, las instituciones, en mayor o menor medida, se han afanado por proteger este legado patrimonial, pero a pesar de la importancia de esta tarea, el modo en que se hace desde los organismos no siempre genera beneficios sociales.

De Certau afirmaba que eran estos 'objetos inanimados' los que «organizan en torno suyo la novela de la ciudad» y continúa diciendo que «dan testimonio de una historia

1 «Utopía española del Siglo de las Luces, publicada por vez primera desde 1976, fecha de su edición original. Descubierta entre los papeles del Conde de Campomanes», https://www.circulobellasartes.com/ libros/sinapia/ (Consultado el 28 de junio de 2018). 
que, a diferencia de la de los museos o los libros, ya no tiene lenguaje. En efecto, tienen la función de la historia, que consiste en abrir una profundidad en el presente, pero ya no poseen el contenido (...). Estos objetos salvajes, salidos de pasados indescifrables, son para nosotros el equivalente de lo que eran ciertos dioses de la antigüedad, las 'ánimas' del lugar» (DE CERTAU, 2000, p. 137-138). Es decir, que estos objetos patrimoniales, carentes ya del significado que poseyeron, adquieren hoy día la categoría de divinidad, son lugares que poseen aun, esa aura de la que hablaba Walter Benjamin, a pesar de la 'reproductibilidad mecánica', condicionando nuestras ciudades, no sólo a nivel de desarrollo espacial o de circulación, sino emocional.

Es aquí donde debemos referirnos a la apropiación del espacio, un concepto que se ha trabajado desde una perspectiva marxista y hegeliana, pero también artística, como veremos más adelante. La planificación urbanística, determinada por las medidas de protección patrimonial y ambiental y en gran medida por la economía, promueve, según Martínez, la manipulación de las necesidades y los deseos (MARTÍNEZ, E., 2014). Pero, ¿se apropia la sociedad de su patrimonio histórico? ¿Y de los espacios públicos? En respuesta a la primera pregunta, debemos referirnos a Riegl, quien defendió que el monumento histórico se construye como tal a posteriori, a través de historiadores y aficionados que seleccionan de entre todos los edificios, una pequeña porción que considera significativos y que a partir de ese momento adquiere nuevos valores de significación y a veces, de uso (RIEGL, 2017).

Son esos testimonios del pasado, monumentos -etimológicamente ya nos indica lo que son, recuerdos-, los que adquieren una concepción filosófica que pone en relación el pasado, el presente y el futuro, de manera lineal; su existencia, como señala Choay, es universal, incluso en aquellos pueblos que no conocen la escritura (CHOAY, 2001), pero los valores mutan. En la actualidad, el patrimonio presenta valores ligados no sólo a la identidad y al memento, sino al ocio, al disfrute e incluso al desarrollo económico a través del turismo. Es aquí donde las instituciones instrumentalizan el patrimonio que termina por desligarse de la sociedad en la que se inserta, para convertirse en un objeto de consumo y produce, en palabras de Arantes, la elitización de las áreas históricas (ARANTES, 2016) y por consecuencia, la gentrificación, tema que se abordará más adelante.

Frente a esto, surgen otros modelos de protección patrimonial, donde se intenta evitar la gentrificación mediante la rehabilitación de áreas más turísticas al mismo tiempo de otras que lo son menos, fomentando además la participación colectiva. Ejemplo de ello son los casos de Bolonia y Oporto, que en la rehabilitación de sus centros históricos han promovido la sostenibilidad del patrimonio y la reapropiación social evitando su musealización y promoviendo que sean espacios vivos (DA ROSA, 2017).

\section{EL ESPACIO URBANO DE LA CIUDAD ACTUAL. ALGUNOS PROBLEMAS}

En 2015, cerca de 4000 millones de personas (el 54\% de la población mundial) vivía en ciudades y, según las proyecciones, ese número aumentará hasta aproximadamente 
5000 millones para 2030 (WEB ONU). Esta rápida urbanización comporta perjuicios como que crezcan los barrios marginales y mayor contaminación atmosférica, entre otras cosas. Es por ello, que uno de los objetivos de la ONU es precisamente mejorar la gestión urbanística para lograr ciudades más sostenibles, resilientes y seguras. Por ello, de aquí a 2030, proponen aumentar las políticas participativas e inclusivas en la planificación urbana, así como en la salvaguarda del patrimonio cultural y natural. (WEB ONU)

Uno de los primeros problemas que presenta la ciudad contemporánea, es la necesidad de aumentar el acceso universal a zonas verdes y espacios públicos seguros, tal y como se desprende de la Conferencia Hábitat III celebrada en Quito (Ecuador) en 2016 y la Nueva Agenda Urbana que surge de ella (WEB ONU-Hábitat). Lotta define el espacio público «como la esencia de la ciudad, la primera motivación de la existencia de un lugar, en la cual el hombre, como parte de una comunidad, vive y se relaciona: la columna vertebral de la ciudad» (LOTTA, 2010, p.63), y se retrotrae para ello al que considera el espacio público por excelencia, el ágora griega, y desde ahí, las plazas, donde los individuos eran ciudadanos y donde se encontraban los edificios de usos comunes, el lugar donde la sociedad se reconocía. Según Lotta, esto cambia con el sistema capitalista, cuando se refuerza la idea del individuo, de manera que los lugares de relaciones se trasladan a edificios privados y cerrados, como los centros comerciales.

A nivel normativo europeo, es la Carta de Leipzig sobre Ciudades Europeas Sostenibles (aprobada con motivo del Encuentro Informal de Ministros sobre Desarrollo Urbano y Cohesión Territorial celebrado en Leipzig en el año 2007), la que regula el desarrollo de la ciudad contemporánea. La UE pretende definir un modelo urbano más sostenible, versátil, eficiente y accesible (LIBRO BLANCO, p.14). En España, se materializa a través del Libro Blanco de la Sostenibilidad en el Planeamiento Urbanístico Español que tiene como finalidad servir de guía (WEB FOMENTO ESPAÑA), a pesar de la heterogeneidad del país y el trasvase de competencias, pues es la municipalidad quien se encarga de la planificación urbanística a través de los Planes Generales (PGOU). El Libro Blanco propone un decálogo en cuyo punto 7 destaca la importancia de la participación ciudadana.

Otro de los problemas es que los actuales modelos urbanos están centrados en el automóvil, lo que genera consecuencias negativas, como congestión, problemas de infraestructura, contaminación (ONU Hábitat) y por supuesto determinan un uso de la ciudad por parte de los ciudadanos que antepone la circulación a las relaciones y apropiación de los espacios. El Fondo de Población de las Naciones Unidas (UNFPA) y ICLEI «Gobiernos locales por la sostenibilidad», así como otros organismos internacionales y locales, se están preocupando por los efectos negativos del tráfico rodado a nivel medioambiental y de salud, incluso de los perjuicios que causa en la conservación de los monumentos, que ha conllevado a la peatonalización generalizada de centros históricos, sin embargo, se tratan poco los perjuicios sociales a nivel psicológico y de relaciones.

Algunos teóricos sí se han preocupado por «encontrar el momento de instaurar una nueva habitabilidad más creativa, más sincera, a partir de la cual hacer que los espacios colectivos adquieran un nuevo sentido para la vida de sus habitantes» (MORENO; MÁR- 
QUEZ, 2011, p.40), como Jaime Lerner quien creó el concepto de acupuntura urbana, definido como cualquier actuación que produzca efectos positivos en la ciudad, favoreciendo un modelo de urbe en la que tiene prioridad la equidad, la convivencia, la cohesión social, la cultura y la educación (LERNER, 2010).

El tercer problema que quisiera tratar sobre la ciudad contemporánea es el de la gentrificación, «la transformación de un área de clase trabajadora de la ciudad en una zona de clase media, para su uso residencial o comercial» (SEQUERA, 2015, p. 2). El sociólogo Daniel Sorando y el urbanista Álvaro Ardura, partiendo de Manhattan, recorren en su ensayo diversas ciudades, explicando este proceso que surge con la reconversión de espacios industriales. En su libro, plantean si es posible mejorar las condiciones de los centros históricos sin cambiar su composición social. Definen además cinco fases en el proceso: abandono, estigma, regeneración, mercantilización y resistencia (SORANDO, ARDURA, 2016). La profesora de sociología Sharon Zukin, explica como los inversores emplean la industria cultural para atraer capital (ZUKIN, 1987).

Es decir, la industria cultural, generalmente desde las instituciones, está provocando en muchas de nuestras ciudades procesos de gentrificación que se ven intensificados por otros movimientos especulativos como los ABNB, cuya consecuencia es el abandono de los centros históricos por parte de sus habitantes habituales, y sus comercios tradicionales, la desaparición de la vida normal y cotidiana, en favor de la ocupación temporal turística y negocios orientados hacia este tipo de público. Además del factor social, hay que tener en cuenta el deterioro que esto provoca en las ciudades a nivel cultural, la pérdida de tradiciones y de identidad. Los centros históricos se homogenizan estética, gastronómica y socialmente en pro de quien busca un selfie para subir a redes sociales. Esto no significa que debamos demonizar el turismo, la posibilidad de viajar se ha democratizado y esto es positivo, un mayor número de personas pueden conocer in situ el patrimonio mundial y no debemos olvidar que es una fuente importantísima de riqueza, un motor económico básico para países como España o Italia, entre otros. Pero debemos, no obstante, analizar también los efectos negativos, que conllevan incluso la pérdida de identidad por parte de la población local que deja de identificarse con aquellos lugares que ya no habita porque han sido "tomados" por el visitante. Hay que tener en cuenta también que esto mismo puede comportar, en un futuro no muy lejano, al agotamiento del sector.

\subsection{Propuestas institucionales y respuestas sociales}

¿Qué respuestas proponen las instituciones a nivel local a estos problemas? En muchos casos, pocas. De hecho, suelen ser responsables de ellos. Hemos mencionado tres problemas fundamentales, la necesidad ciudadana de apropiarse de los espacios libres; los canales de circulación, pensados para el tráfico rodado y la gentrificación. En cuanto al primer problema, es evidente que las ciudades no están pensadas para el peatón y que el crecimiento de las ciudades provoca la pérdida de espacios libres o zonas verdes. Generalmente, este problema se viene solucionando colocando mayor número de zonas 
abiertas, parques o paseos, en los nuevos barrios construidos a las afueras de las ciudades, barrios dormitorios que se convierten en un pequeño pulmón para la ciudad, pero que obligan a quienes no habitan en ellos a desplazarse a una zona lejana para poder disfrutar de espacios de relación.

Frente a esto, surgen iniciativas sociales que sí promueven la apropiación del espacio por sus gentes, desde asociaciones de vecinos. Por ejemplo, en Palermo, algunos jóvenes comenzaron a preocuparse activamente por la relación que tenían con el espacio público, logrando la constitución del Comitato Spazio Pubblico, un grupo que comenzó a «invadir» un área simbólica de la ciudad, pero ahogado por coches que lo cruzan y que aparcan allí cada día: la Piazza Bellini, dejando de manifiesto que la población pide más espacio público (LOTTA, 2010, p.64).

En este sentido podríamos hablar de la tendencia a crear huertos urbanos y jardines secretos, desde el Parco Nord de Milán, a cualquier ciudad periférica. Según el diario La Vanguardia «en España hoy existen más de 508 huertos urbanos. Un número que ha crecido desde el año 2000 en un 98\%» (NAVARRO, R., 2016). Entre sus beneficios se mencionan cuestiones como el fomento de las relaciones sociales o la sostenibilidad. De alguna forma, se intenta introducir lo rural en lo urbano, volver al contacto con la naturaleza, a esa vida nómada perdida, pero sin perder el contacto con el asfalto y el sedentarismo. No obstante, también comienzan a emerger voces críticas, por el alto grado de polución presente en los productos, entre otras cosas. Estas iniciativas, que a veces parten de los vecinos, se han institucionalizado y son muchos los ayuntamientos que sortean estas parcelas en diversas localidades, creando un auténtico furor (MONTAÑÉS). En ocasiones, no sólo los encontramos en grandes urbes como Barcelona, sino también en poblaciones más pequeñas que realmente no han perdido el contacto con el medio rural, como puede ser el caso de Úbeda (Jaén) y los huertos de la Fundación Huerta de San Antonio, cuyos productos pueden venderse en un mercado celebrado en la antigua Iglesia de San Lorenzo, hoy con uso cultural, en una integración perfecta de lo que venimos tratando, recuperación del patrimonio y de los espacios, sin provocar gentrificación, sino integrando a los habitantes.

En cuanto a los jardines secretos, típicos en ciudades como Londres, Roma o Milán y presentes por ejemplo en Madrid, no son sino espacios semi comunales-semi públicos, zonas verdes para relación de los vecinos, pequeños pulmones de las grandes urbes. Sin embargo, una nueva tendencia propone crear estos jardines secretos en centros históricos con solares no urbanizados, con la idea de recuperar estos espacios, muy degradados. Un ejemplo es la ciudad de Jaén, que cuenta con un centro histórico repleto de solares, por lo que el consistorio se planteó crear una red de jardines secretos. Hasta la fecha se ha inaugurado uno («Al jardín secreto...»), pero está vallado, por lo que sí, se ha conseguido mejorar la imagen y la salubridad del lugar, pero no se le ha ofrecido a la ciudadanía un nuevo espacio de relación.

Por otro lado, la preminencia del tráfico rodado provoca que en ciertas zonas de las ciudades, los peatones se salten con frecuencia los caminos determinados e incluso que incumplan las normas viales. Así surgen los llamados caminos del deseo, recorridos que 
la ciudadanía, en un acto de rebeldía, impone en la ciudad sobre las vías prestablecidas, que no están pensados para el caminar. Esas líneas del deseo, acuñadas por el ya citado Bachelard, son el reflejo de una decisión consciente de querer tomar una ruta distinta a la prestablecida, dibujando ciudades más humanas. Ante estas nuevas rutas espontáneas, que se consolidan en nuestras ciudades, las autoridades tienden a ignorar el hecho o en ocasiones a multar. Pero existen otras tendencias, en Copenhague el ayuntamiento decidió reconfigurar algunas zonas de la ciudad en función de las líneas del deseo, mejorando así la circulación en la misma (HURST, 2014).

Si nos referimos al problema de la gentrificación, hemos comprobado como en multitud de ocasiones son las propias instituciones las responsables, con esa elitización de los centros históricos y el patrimonio que se convierten en objetos de consumo. Este problema viene dado en numerosas ocasiones por la intención de los organismos locales de aumentar el patrimonio de la ciudad a través del arte contemporáneo, creando museos, centros de arte o rutas de graffiti, en barrios degradados, bajo el pretexto de rehabilitarlos. Pero finalmente lo que se produce es un aumento de la especulación y por consecuencia, la gentrificación del barrio, que se torna turístico y para la élite.

En general, las instituciones locales han estado preocupadas por ofrecer nuevas etiquetas a la ciudad contemporánea, con apelativos como «ciudad del conocimiento, smart city, ciudad informacional, ciudad en red, etc.» (RODRÍGUEZ; CALO; OTERO, 2017, p. 700), promoviendo el acceso a wifi en las calles o soluciones ante la contaminación. Dentro de toda esta terminología, en A Coruña surge uno diferente, el de "ciudad creativa", «un ejemplo de instrumentalización cultural» (RODRÍGUEZ; CALO; OTERO, 2017, pp. 704-705).

Por otro lado, hay que destacar la utilización de lo efímero mediante la creación de eventos de arte contemporáneo que se suman a las fiestas tradicionales. En la ciudad del espectáculo, eventos como las exposiciones universales, ferias de arte o diseño, o incluso eventos deportivos como mundiales, u olimpiadas, entre otros, promueven que las instituciones se afanen por conseguir celebrar uno de estos eventos para así atraer una vasta cantidad de personas que se concentran en las ciudades por una o dos semanas. Incluso otros eventos como las shopping night o el black Friday, ligados al comercio, promueven la aparición de manifestaciones artísticas en la calle con carácter efímero: graffiti, arquitectura efímera, música, teatro, etc.

El paradigma sin duda es la creación de La Noche en Blanco, una iniciativa cultural creada en París en 2002, cuyo gran éxito ha promovido que se extienda a otras ciudades del mundo. El objetivo de la Noche en Blanco es acercar el arte contemporáneo y la creación artística a los ciudadanos, pero en ocasiones se ha ido desvirtuando y en otros se ha convertido en otro punto más para la atracción turística.

Por otro lado, también encontramos la elección de la capital gastronómica o la capitalidad europea de la cultura, entre otras «fiestas efímeras». Cuando en 2016 San Sebastián fue elegida Capital Cultural, otra candidata que se postuló fuertemente para ser elegida, Córdoba, había dedicado una década a gestar el proyecto que contaba con nada 
menos que 113 millones de euros (EUROPAPRESS). Otras iniciativas en este sentido puede ser la colocación de las Meninas en Madrid $^{2}$, que ha generado un movimiento ciudadano para que se retiren.

\subsection{Propuestas artísticas}

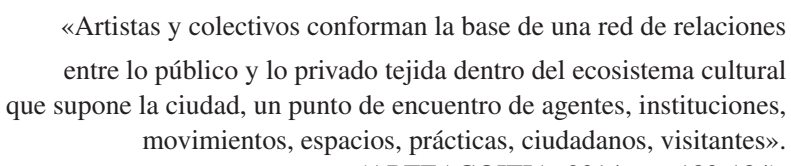

(ARTEAGOITIA, 2014, pp. 183-184).

Si bien arquitectura y urbanismo habían dejado nulo margen de opinión con respecto a la construcción de los espacios públicos a los artistas (SZMULEWICZ, 2017, p. 4), las artes plásticas siempre se han sentido atraídas por la representación de las ciudades, desde vistas a planimetrías. Es innegable la relación entre la pintura de Mondrian con las maquetas de Van Doesburg, por ejemplo. Incluso el Arte Pop tuvo un compromiso con el entorno, como se aprecia en la obra de Oldenburg, esos grandes objetos instalados en las ciudades (RODRÍGUEZ PEDRET, 1991). Más reciente es la obra de Rachel Whiteread (Londres 1963), a través del grabado que «explora las conexiones que existen entre la arquitectura, el espacio natural y las personas» (ARTEAGOITIA, 2014, pp.186-187). Miguel Ángel Moreno Carretero también se interesa por los objetos de la ciudad, con proyectos como «Mecanismos inconscientes del horizonte', «Paisajes disimulados», «Paisajes preferentes» (MORENO CARRETERO, 2014) o más recientemente en «Ofrendas», maquetas de edificios ligados al poder político y económico, que simulan esos edificios en manos de santos que promovieron grandes construcciones eclesiásticas que se representan tradicionalmente en pinturas y esculturas. Moreno nos ofrece estas maquetas como los nuevos monumentos de la ciudad contemporánea, para que todos podamos situar la mano debajo y sostener ese pedacito del patrimonio o simplemente, sostener el poder.

Frente a la necesidad de apropiarse de los espacios, algunos artistas están optando por las acciones. Capelata, Marzulo y Carrero, explican como «si históricamente la ciudad como locus de la experiencia urbana tiene como una de sus características ser polo de la producción artística, (...) también trae en su configuración, la ocupación de espacios públicos por acciones artísticas» (CAPELATO, MARZULO, CARRERO, 2017, p.314). Por ejemplo, Aida Navarro, arquitecta, concibe el espacio público como tablero de juego, creando así ficciones por aproximaciones, como estrategia para abrir posibilidades. (TEDX UPValencia).

También, como herencia del Situacionismo, encontramos el caminar como forma de apropiación, «la caminata, como investigación del espacio urbano, presente en el de-

2 Meninas Madrid Gallery, Antonio Azzato (2018). 
sarrollo de las culturas primitivas, ha sido olvidada por los arquitectos y los urbanistas, han sido los poetas y los artistas plásticos quienes han intentado recuperarla. Con esta práctica se atribuyó un valor estético al espacio» (NAVARRO FUENTES, 2016, p. 118). En este sentido, el artista malagueño Antonio R. Montesinos, ha realizado experiencias relacionadas con la deriva, como el proyecto «Ruta ultrapotásica»o «Perimetrías», entre otras. En esta última, propone guías para el paseo a través de Google Maps, con la idea última de alejarnos del utilitarismo urbano.

Pero en cuanto a apropiación del espacio, hay que mencionar sin duda a colectivos como Boa Mistura, Basurama, o Truth Behind 404. El primero, con dos dimensiones a través de los muros, retoma esa idea de la acupuntura urbana a través de mensajes positivos. Por otro lado, Basurama recupera espacios degradados mediante la creación de autobarrios. Truth Behind 404, propone acciones como Make a city, a través de Medialab Prado, donde crea un banco que puede imprimirse con una impresora en 3D, para que quien quiera lo coloque en una zona de la ciudad en la que se haya perdido ese espacio público, únicamente en pro de las terrazas de los bares. Lo que tienen en común todos ellos es que ponen en práctica el concepto de arte relacional de Bourriaud, integrando en sus proyectos a los habitantes de los barrios en los que intervienen, de manera que indicen en ellos, sintiéndose identificados con las creaciones y re-apropiándose de un espacio que realmente les pertenece. Beuys, con su concepto de 'arte ampliado', fomentó la integración del arte y la vida. En este sentido, López Aparicio afirma que «es fundamental que exista un equilibrio entre los objetivos propios del artista, que pretende proyectarse en su nicho de actividad genuina, y las necesidades, la dignidad, de la comunidad con la que, y desde la que, se trabaja» (LÓPEZ APARICIO, 2016, 24).

Nanna Verhoeff, trata sobre la dramaturgia de las ciudades a través de instalaciones como 'In the Air, Tonight', un proyecto de los artistas Davila y Colangelo, con vocación efímera y tecnológica, que consiste en usar Twitter para, a través del hashtag \#homelessness, cambiar las proyecciones azules sobre el edificio Ryerson de Toronto, a color rojo, como una forma de visibilizar el problema de la gente sin hogar en la ciudad (VERHOEFF, 2018). En este sentido, el artista Refik Anadol, a través de las nuevas tecnologías, se apropia de los espacios con performance audiovisuales como «Infinity Room» donde crea mundos no físicos tratando de cambiar la percepción de los espacios en el mundo contemporáneo.

En España, Eugenio Ampudia propone algo similar con intervenciones como «Incendio» (2009) en la que simula fuego en el edificio de Gran Vía 1 (Madrid), inspirado por el Manifiesto Surrealista. También es interesante el concepto del colectivo Luz Interruptus, que con sus instalaciones invaden el espacio con plásticos desechados, para concienciar del problema que supone la acumulación de estos residuos para el planeta.

Realmente, es el arte urbano el que sin duda se ha apropiado del espacio de una manera más real desde su nacimiento. Real porque nace fuera de los márgenes de lo institucional, es un arte incluso prohibido, vandálico, lo que aporta mayor libertad y capacidad crítica, a pesar de los intentos de institucionalizarlo en las últimas décadas a través de la 
creación incluso del primer museo de arte urbano, en Berlín, pero ¿es urbano una manifestación artística que se encuentra dentro de un museo?

En cuanto a los arquitectos, son sin duda los asiáticos quienes están proponiendo un concepto más novedoso, preocupados por la sostenibilidad, el medio ambiente y la circulación. Ejemplo de ello es Ma Yansong que propuso en los años '80 el concepto de 'ciudad Shanshui', con la idea de volver a la naturaleza sin salir de la metrópoli, si bien todo quedó en el plano teórico. Pero más interesante aun es la labor de 100 Architects con sede en Shangai, realizan intervenciones urbanas con objetos arquitectónicos, tanto efímeros como permanentes, que buscan la interactuación y la estimulación de la dinámica social.

\subsection{Una visión de género}

Otro de los grandes problemas de las ciudades es la falta de seguridad para las mujeres. Las instituciones se centran en aumentar la vigilancia y en algunos casos, se está empezando a optar por servicios públicos de transporte nocturno sólo para mujeres. ¿Pero es esta la mejor y única vía? La ONU advierte de que «las mujeres y los hombres, las niñas y los niños, experimentan el proceso de urbanización y la vida en la ciudad de manera diferenciada» (WEB ONU Hábitat). La ausencia de mujeres en puestos de gobierno ciudadano dificulta la visión inclusiva de las ciudades; por otro lado, destacan los «diseños urbanos pobres, como calles con mala iluminación y pasajes subterráneos solitarios» (WEB ONU Hábitat).

Queda claro que las planificaciones urbanas necesitan de un enfoque de género. Una buena forma de hacer la ciudad más igualitaria es mediante la incorporación de mujeres arquitectas y urbanistas (RODRÍGUEZ CALATAYUD, 2008); en España han surgido colectivos que proponen cambiar el modelo de ciudad para que esté pensada para ser vivida y no sólo para moverse y trabajar, como Mujeres Urbanistas o la Fundación María Aurelia Campmany (ORTIZ GUITART, 2007).

Además de esta visión urbanista, volvemos a recalar en la deriva como forma de apropiación de los espacios. Laura Elkin (ELKIN, 2016) retoma el término flâneuse, usado por Janet Wolf como variante de flâneur, paseante callejero, que sólo tiene versión masculina, en referencia a las mujeres que cambiando el modo de moverse por el espacio urbano, pueden intervenir en la organización del mismo. Andrea Burbano, propone también la apropiación del espacio urbano como una forma de sociabilidad e igualdad de género. Afirma que no podemos asumir el espacio público como neutral al género, puesto que no lo es (TedXUniversidad Piloto, 2014).

Desde el ámbito del arte también se ha reivindicado la ciudad con presencia femenina. La mayoría de las propuestas artísticas entorno al género se centran en acciones, podríamos citar artistas como Verónica Ruth Frías o Yolanda Domínguez. También el caso de Valle Galera, que con su intervención en la plaza Grao de Castellón, en la que pasó un día fotografiando a las mujeres que pasaban, mostró cómo la arquitectura y el espacio público podían influir en su comportamiento y sus movimientos. 


\subsection{El efecto del nuevo museo}

En este recorrido por la ciudad contemporánea, sus problemas y las soluciones ligadas al arte, no podemos dejar al margen la cuestión de los grandes proyectos constructivos, ligados con frecuencia a la cultura y especialmente al arte contemporáneo. Un ejemplo ilustrativo de cómo las ciudades tienden a la homogeneidad por las políticas constructivas y de atracción turística, serían las norias. El London Eye, una noria de 135 metros inaugurada en el año 2000, generó un efecto imitación en Europa que provocó que todas las capitales quisieran su propia noria. La de París, abierta en el mismo año, podría no volver a montarse el próximo año. La prensa ya hablaba de un símbolo de la ciudad, pero ¿puede un elemento festivo como una noria, presente desde hace menos de dos décadas convertirse en un símbolo de una ciudad que cuenta con monumentos tan emblemáticos como la Torre Eiffel, el Louvre, los Campos Eliseos, el Arco del Triunfo... e incluso Disneyland? ¿O es una campaña de marketing en la que hay que incluir algo nuevo para recordar que la ciudad está ahí?

Sí encontramos una noria emblemática en Viena, la Riesenrad, inaugurada en 1897 con motivo del aniversario del reinado de Francisco José y situada en este caso en un lugar que parece apropiado, un parque de atracciones. Si buscamos en internet qué hacer en Budapest, entre las cinco cosas más destacadas nos aparece subir a la Budapest Eye, noria a imitación de la londinense. Y así con tantas y tantas ciudades del mundo como Los Ángeles, Singapur, Chicago, Tokio, etc. En España, encontramos una en Barcelona, situada en el parque de atracciones del Tibidabo y con una antigüedad que se remonta a 1953. Otra gran noria la encontramos en el Puerto de Málaga, y que en estos momentos está en proceso de ampliación. En 2017 un proyecto de montar en Madrid una noria como la de Londres se quedó en un cajón, necesitaba una inversión de 20 millones y pretendía convertirse en emblema de la ciudad. En el Muelle de las Delicias de Sevilla se instaló una noria que duró quince meses, tras haber desembolsado siete millones de euros ${ }^{3}$.

Sin duda, estos dos últimos ejemplos ilustran perfectamente en qué se convierte la moda de la noria, homogeneiza las ciudades, por lo que ya no es un símbolo reconocible de ninguna ciudad a pesar de que sea esa la pretensión; no son sostenibles, la inversión no se recupera fácilmente, ya que las entradas son elevadas; en muchas ocasiones se sitúan en lugares inadecuados, alejados de las zonas turísticas o sin vistas y lo que es peor, en ciudades que tienen otro tipo de miradores más atractivos; y por último, suelen relacionarse con atracciones de feria y no como elementos permanentes. Sin embargo ¿por qué

3 Para ampliar información sobre las norias se pueden consultar algunos titulares de prensa como los siguientes: https://elviajero.elpais.com/elviajero/2018/03/29/album/1522335000_800182.html\#foto_ gal_23 / https://www.elconfidencial.com/espana/2017-10-21/london-eye-madrileno-inversion-20millones-rechaza-carmena_1464216/ / https:/www.laopiniondemalaga.es/malaga/2017/09/21/noriapuerto-recibe-nueva-prorroga/956920.html / http://sevilla.abc.es/sevilla/sevi-noria-sevilla-seis-razonespara-fracaso-201610141856_noticia.html / http://sevilla.abc.es/sevilla/20141003/sevi-noria-sevillapuerto-201410031345.html (Consultadas 29 de junio de 2018) 
continúa la fiebre de las norias? Esta tendencia sería comparable a la carrera por construir el edificio más alto ${ }^{4}$, no hay que irse ya a los grandes rascacielos de EE.UU o más recientemente Asia, sino comprobar la polémica que supuso en Sevilla la construcción de la Torre Sevilla (conocida como Torre Pelli), otra torre, como la de Bilbao (Torre Hiberdrola), construida por el autor de las famosas Torres Petronas de Kuala Lumpur5. Para algunos, un atentado contra la ciudad puesto que supera en altura a la Giralda, para otros un símbolo del avance hacia la contemporaneidad, junto con el famoso Metropol Parasol (las setas), de la plaza de la Encarnación, en pleno centro histórico, también polémico tanto por la estética, como por doblar el presupuesto inicial.

Esta moda que ilustra perfectamente la tendencia actual de las instituciones municipales, a crear productos de consumo en las ciudades donde se pierde el carácter propio de cada población, pretende crear de forma forzada nuevos elementos de identificación más hacia el exterior que hacia la propia población local y por supuesto alejados de la sostenibilidad, puesto que mientras se invierten ingentes cantidades en esto, se abandonan otros elementos patrimoniales. A esto habría que sumar las causas del llamado Efecto Guggenheim. Una ciudad, y en especial una zona, muy degradada y deprimida en un momento determinado, Bilbao en los años '80, se renovó gracias, entre otras cosas, a la instauración de un museo de arte contemporáneo, para el cual se eligió a un arquitecto enormemente conocido y monumental, Gehry. Este edificio se convierte, este sí, en un símbolo de la ciudad contemporánea, en diálogo con la ciudad histórica al otro lado del río y con el museo de la ciudad, a pocos metros.

Tras esto, muchas ciudades quisieron sumarse a la moda del nuevo museo, un centro de arte contemporáneo situado en un gran edificio que sirviese para renovar la imagen de la ciudad y para reavivar o regenerar barrios - provocando en muchos casos la temida gentrificación-. Pronto se sumó León, con el MUSAC, un centro de arte contemporáneo cuyo edificio fue erigido por los arquitectos Mansilla y Tuñón y que recibió el premio Mies van der Rohe de 2007, un referente cultural en la comunidad de Castilla y León que se sumaba a uno anterior, el Museo Patio Herreriano de Valladolid, ambos, según datos oficiales, con cifras de visitantes muy similares ${ }^{6}$, superando incluso el número de visitas de algunos monumentos históricos, y promovido en parte por el efecto reclamo de las exposiciones «Las edades del hombre» ${ }^{7}$. Otro ejemplo lo encontramos en Cantabria, con la reciente creación del Centro Botín, en un edificio diseñado por Renzo Piano, buscando

$4 \quad \mathrm{Y}$ otras grandes inversiones como los aeropuertos fantasmas.

5 Y autor de otras tantas torres similares como la YPF de Buenos Aires, la Torre de Cristal de Madrid, etc.

6 Datos estadísticos sobre el MUSAC en Portal de Museos de Castilla y León. file://C:/Users/laura/ Desktop/Por\%20ver/Ep\%C3\%ADgrafe+13_8.b.pdf

Datos estadísticos sobre el Patio Herreriano en Portal Ayuntamiento de Valladolid. https://www. valladolid.es/es/actualidad/noticias/museo-patio-herreriano-suma-casi-70-000-visitantes-2017-sup (Consultadas 29 de junio de 2018)

7 Cifras de 2010 ofrecidas por El Mundo. http:/www.elmundo.es/elmundo/2010/06/12/ castillayleon/1276332782.html (Consultada 29 de junio de 2018) 
nuevamente la espectacularidad del contenedor, si bien la diferencia es que este es un centro privado, que no ha requerido inversión pública. El caso contrario sería la Ciudad de las Artes y las Ciencias de Valencia, de Calatrava y Candela, con unos sobrecostes de 625 millones de euros del erario público. Otro caso contrario al espíritu del Efecto Guggenheim, pero no por cuestiones económicas, sería el MACBA de Barcelona, situado en pleno centro histórico, con gran arraigo, pues se inauguró en 1995, asiste ahora a la degradación del Raval.

Este caso no obstante es interesante porque se sitúa dentro del centro histórico, a diferencia de otros como el MUSAC que están en barrios nuevos. ¿Cómo se integra lo contemporáneo en la ciudad histórica? No exento de polémica. Muchos son los detractores de este tipo de prácticas, también en ciudades más periféricas como Jaén, donde la creación del Teatro Infanta Leonor, en los límites del centro histórico, también muy degradado, creó un gran número de escépticos puesto que el edificio presentaba una estética, como no podía ser de otra manera, contemporánea. En Andalucía hemos asistido a este fenómeno a través de la creación de los Centros Andaluces de la Danza, de las Letras, del Teatro y especialmente de la Fotografía en Almería, y de Arte Contemporáneo, este último con su versión sevillana y malagueña, iniciados en la década de los '90 y actualmente infradotados en algunos casos, aunque con gran autonomía y éxito de público y crítica en otros, como es el caso de Málaga ${ }^{8}$ y más recientemente con el Museo Íbero de Jaén.

Según cifras del Ministerio de Cultura9 ${ }^{9}$, los museos de arte contemporáneo son los que más visitas reciben, sólo superados por los de bellas artes, según datos de 2016; siendo el número de extranjeros importante, pero no determinante, lo que indica una asistencia importante de personas locales, a pesar de que en el Guggenheim un 64\% de los visitantes sí son foráneos ${ }^{10}$. Después de Madrid y Cataluña, Andalucía es la comunidad que más visitantes recibe en sus museos. En Andalucía, los museos más visitados son de bellas artes, en concreto los de Granada y Sevilla, junto con el Museo de la Alhambra, pero la cifra del Centro Andaluz de Arte Contemporáneo (CAAC) es mucho más elevada que la del resto de museos públicos. Podemos concluir que el turista que viene a Andalucía busca la tradición por encima de la contemporaneidad a pesar de los esfuerzos por instaurar este tipo de centros para reforzar la oferta turística y modernizar la imagen de las ciudades, si bien el número de visitantes que buscan lo contemporáneo no es nada desdeñable ${ }^{11}$.

$8 \quad$ No exento de polémicas por su dirección, sobre todo entre los artistas de la zona.

9 https://www.mecd.gob.es/servicios-al-ciudadano/estadisticas/cultura/mc/culturabase/museos-ycolecciones-museograficas/resultados-museos/2016.html (Consultado el 29 de junio de 2018)

10 Dato publicado por El Mundo. http://www.elmundo.es/pais-vasco/2015/01/02/54a68d84268e3e612f 8b4582.html (Consultado el 29 de junio de 2018)

11 Datos estadísticos proporcionados por la Consejería de Cultura de la Junta de Andalucía. http://www. juntadeandalucia.es/organismos/cultura/consejeria/sobre-consejeria/estadistica-cartografia/paginas/ estadisticas-museos-badea.html (Consultado el 29 de junio de 2018) 
En este sentido Málaga es el paradigma, una ciudad con un patrimonio histórico rico, pero ensombrecido por grandes monumentos cercanos como los que componen la oferta turística de las vecinas Granada, Córdoba o Sevilla, se ha afanado en las últimas décadas por ligarse a la creación contemporánea a través no sólo de la citada Noche en Blanco o el CAC, sino también el Museo Picasso, la sede del Centro Pompidou, la sede el Museo del Hermitage, o el Carmen Thyssen, entre otros, además de la creación del $\mathrm{SOHO}$, el barrio en torno al CAC, donde se ha fomentado el graffiti y se ha producido la elitización y gentrificación. Habría que sumar otras propuestas más alternativas como La Térmica. A pesar del aparente éxito de estas iniciativas culturales, el número de visitantes, y sobre todo que paguen entradas, es muy inferior al esperado ${ }^{12}$. La creación de «franquicias» de los museos también es una moda actualmente, no hay más que echar un vistazo a la nueva sede del Louvre en Abu Dabi, diseñada por Jean Nouvel. También en Andalucía, pero en este caso en la ciudad de Córdoba, encontramos un ejemplo contrario, las diversas salas de exposiciones que se centran en el arte actual, pertenecientes a distintas instituciones, se dirigen sobre todo a un público local o a un turista más especializado y promueven especialmente a los artistas jóvenes de la provincia o la comunidad, además de mostrar a algunos foráneos, son los casos de la Casa Góngora, la Galería Vimcorsa, el Centro Botí, Modo o el Espacio Iniciarte, entre otros, dejando a la masa de turistas las visitas a los lugares típicos que constituyen la marca Córdoba como la Mezquita, la Sinagoga y demás. Además, la provincia cuenta con un gran número de propuestas desde lo local y lo rural en pro de lo contemporáneo, ligado al contexto y los habitantes locales, como Scarpia, Dmencia, Z o Art Sur, etc.

Un buen ejemplo de integración de los espacios dedicados al arte contemporáneo en una ciudad rica en patrimonio histórico es el de Roma. Posiblemente la ciudad con mayor cantidad de patrimonio de todas las épocas, ha sabido crear espacios como el MACRO y el MAXXI, nuevamente, sobre todo en el caso del segundo, optando por una arquitectura espectacular de mano en este caso de Zaha Hadid. Eso sí, ambos, sobre todo el MAXXI, muy alejados de las zonas turísticas. Según el ISTAT, en Italia los museos reciben más visitas que las áreas arqueológicas y monumentos ${ }^{13}$, no obstante, entre los treinta museos más visitados de Italia no hay ninguno contemporáneo ${ }^{14}$. En concreto, el MAXXI recibe más visitas que el MACRO, ambos muy por delante de otros espacios de arte no contemporáneo como el Palacio Barberini ${ }^{15}$. Si bien en Italia no recogen los datos de procedencia

12 Sobre este tema se recomienda la crítica que hace Rogelio López Cuenca en 2018 «En la ciudad (,) donde la cultura es capital», Actas $3^{\circ}$ Congreso Internacional Arte, Ciencia y Ciudad, pp. 37-48.

13 Datos de 2015. ISTAT. I musei, le aree archeologiche e i monumenti in Italia.

14 Datos del Ministero dei Beni e delle attività culturali e del turismo. Referentes a 2017. http://www. beniculturali.it/mibac/export/MiBAC/sito-MiBAC/Contenuti/visualizza_asset.html_249254064.html (Consultado el 29 de junio de 2018).

15 Datos de 2014 recogidos por Federculture. www.federculture.it/.../Dati-ROMA-e-LAZIO_RapportoFedercult Y datos 2016, 2015 de Comune di Roma. https:/www.comune.roma.it/web-resources/cms/ documents/Cultura_2016_REV.pdf (Consultado el 29 de junio de 2018). 
de los visitantes, parece claro que son museos más visitados por locales, especialmente por sus exposiciones temporales y otros eventos. Refuerza esta idea el hecho de que los visitantes que pagan entrada en estos museos son muy escasos ${ }^{16}$.

Este ejemplo nos sirve para ilustrar como lejos de intentar competir en significación con elementos patrimoniales con una gran identidad, en Roma, y en general en Italia, han optado por un modelo en que estos nuevos espacios complementan la oferta turística, sí, pero una oferta para un público especializado y sobre todo tratan de fidelizar a los visitantes locales que puede disfrutar de estos lugares, no hay más que pasear por el entorno del MAXXI para comprobar como su plaza delantera sirve de espacio de socialización para los habitantes del barrio.

\section{CONCLUSIONES Y PROPUESTAS}

«Cuando el que reside en la ciudad siente que forma parte de un lugar, de unas relaciones sociales y goza de la ciudad, es entonces cuando aporta y recibe del entorno mucho más de lo que cualquier documento de planificación pueda prever, dictar e incluso imaginar». (MORENO; MÁRQUEZ)

En este texto se ha tratado de abordar la ciudad contemporánea desde distintas perspectivas para entender algunos de sus problemas actuales más significativos, así como atender a las soluciones propuestas desde las instituciones y desde la creación artística. Lo cierto es que la ciudad histórica se integra en la ciudad contemporánea como un área de respeto, en ocasiones sobre explotada, que provoca su elitización y la falta de identificación por parte de los habitantes provocando la especulación y por consiguiente la gentrificación. La ciudad actual se muestra en cierto modo deshumanizada por la pérdida de espacios públicos para relacionarse y por la preponderancia de la circulación a motor por encima del tránsito a pie.

Asimismo aparecen problemas derivados de la falta de seguridad, especialmente para las mujeres; y de contaminación, estos últimos quizá los más tratados por las instituciones. Frente a esto, los artistas proponen soluciones que sirven para hacer reflexionar o incluso para apropiarse del espacio urbano, si bien no son el remedio para un problema global que seguirá en aumento en las próximas décadas según los datos oficiales. Por otro lado, encontramos la idea de la gran construcción, de la espectacularidad de la arquitectura, muchas veces ligadas al arte contemporáneo, para atraer público y bajo la excusa en ocasiones de regenerar barrios o crear nuevas identidades de la ciudad, pero que suponen grandes deudas con demasiada frecuencia.

Queda claro que el Efecto Guggenheim no es universal, no siempre sirve para regenerar espacios colocar un nuevo espacio cultural y no siempre crean identidad, si bien

16 Según Francesco Pellegrino en el libro «MAXXI. Cronache dal museo: i primi cinque anni» (Campisano: 2016) 
debemos seguir cuidando el turismo como motor económico, debemos repensar las ciudades y su patrimonio para lograr una mejor integración entre lo nuevo y lo viejo y entre el habitante y el visitante, evitando la homogeneización de las ciudades por modas.

En todo esto, los historiadores del arte debemos también jugar un papel importante en cuanto a describir las ciudades más allá de su morfología y tender entre todos hacia la consecución de urbes más humanizadas, sostenibles y seguras y sobre todo, heterogéneas y participativas. 


\section{BIBLIOGRAFÍA}

«Al jardín secreto del casco antiguo le ha crecido una valla». Hora Jaén [3 de abril de 2018], s.p. https://www.horajaen.com/2018/04/03/al-jardin-secreto-del-casco-antiguo-le-ha-crecido-una-valla/ (consultado el 28 de junio de 2018).

«Sebastián arrebata a Córdoba su sueño de convertirse en Capital Cultural Europea en 2016». Europapress [28/06/2011], s.p. http://www.europapress.es/andalucia/fundacion-cajasol-00621/noticia-cordoba2016-ampl-san-sebastian-arrebata-cordobasueno-convertirse-capital-cultural-europea-2016-20110628174151.html (consultado el 28 de junio de 2018).

Aguilar Rocha, I. S. (2017): «La ciudad contemporánea: identidad y patrimonio», en Actas Cidades Creativas, Icono 14, pp. 97-105.

Arantes, O. B. F. (2016): «Gentrificación estratégica», Arquis, 1, pp. 106-121. (Traducción de Ana Paula Montes).

Arteagoitia García, D. (2014): «La ciudad como matriz. La urbe como pretexto en la gráfica contemporánea», AusArt 2, pp. 181-190.

Bachelard, G. (2000, $4^{\circ}$ ed.): La poética del espacio. Fondo de cultura económica.

Beascoechea Gangoiti, J. M.; González Portilla, M.; Novo López, P. A. (2006): La ciudad contemporánea, espacio y sociedad, Universidad del País Vasco.

Burbano, A. (2014): Ideas para una mejor vida urbana, Conferencia TedX Universidad Piloto. https://www.youtube.com/watch?v=960GlVnkTfM (Consultado el 11 de junio de 2018).

Capelato, R.; Marzulo, E.; Carrera, R. (2017): «Espaço público: modos de fazer, modos de usar», en Actas Cidades Creativas, Icono 14, pp. 313-324.

Castro Martínez, P. V.; Escoriza Mateu, T.; Oltra Puigdomenech, J.; Otero Vidal, M.; Sanahuja, E. (2003): «¿Qué es una ciudad? Aportaciones para su definición desde la prehistoria», Scripta Nova, 146, pp. 1-13.

Choay, F. (2001): La invención del monumento histórico. Cambridge Prensa Universitaria.

Conti, A. (2015): «La conservación y la gestión de las ciudades históricas desde la perspectiva del Paisaje Urbano Histórico». Conferencia, 9 de septiembre de 2015. Facultad de Ciencias Económicas, Universidad Nacional de la Plata. http://sedici.unlp. edu.ar/handle/10915/50335 (Consultado el 13 de junio de 2016).

Da Rosa Sampaio, A. (2017): «Centros Históricos de Bolonha e do Porto: lições de reabilitação urbana para o debate contemporâneo», Rev. CPC, São Paulo, 23, p. 40-64

De Certau, M. (2000 ed.): La invención de lo cotidiano. Universidad Iberoamericana.

Debord, G. (1958): Teoría de la deriva. \# 2 de Internationale Situationniste. Traducción extraída de Internacional situacionista, vol. I: La realización del arte, Madrid, Literatura Gris, 1999. http://www.ugr.es/ silvia/documentos\%20colgados/IDEA/teoria\%20de\%201a\%20deriva.pdf (Consultado el 13 de junio de 2018).

Elkin, L. (2016): Flâneuse, Women Walk the City. Penguin Random House. 
Esteban, I. (2007): El efecto Guggenheim. Anagrama.

Fernández Arenas, J. (1988): Arte efímero y espacio estético. Anthropos.

Foucault, M. (1967): «Des espaces autres», conferencia pronunciada en el Centre d'Études architecturales el 14 de marzo de 1967 y publicada en Architecture, Mouvement, Continuité, $\mathrm{n}^{\circ}$ 5, octubre 1984, págs. 46-49. Traducción al español por Luis Gayo Pérez Bueno, publicada en revista Astrágalo, n 7, septiembre de 1997. http://textosenlinea.blogspot.com/2008/05/michel-foucault-los-espacios-otros.html (Consultado el 11 de junio de 2018).

González de Castells, A. N. (2013): «Rehabilitaciones urbanas en la ciudad contemporánea: entre las formas de hacer la ciudad y las formas de hacer en la ciudad», Arxiu d'Etnografia de Catalunya, 13, pp. 217-229.

González Martínez, P.; Tabales Rodríguez, M. A. (2015): «Preexistencias en la ciudad histórica contemporánea: viabilidad y convivencia», $P H$ 87, pp. 202-205.

Heidegger, M. (1994): Construir, Habitar, Pensar. Serbal.

Hurst, M. (2014): «Las líneas del deseo nos ayudan a diseñar ciudades más humanas», Yorokobu. https://www.yorokobu.es/las-lineas-del-deseo/ (Consultado el 16 de junio de 2018).

Lefebvre, H. (1972): La revolución urbana. Alianza.

Lerner, J. (2010, $5^{\circ}$ ed.): Acupuntura Urbana. Editora Record.

López Aparicio, I. (2016): Arte político y compromiso social. El Arte como transformación creativa de conflictos. Cendeac.

Lotta, F. (2010): «Reinventar de los espacios públicos en la ciudad contemporánea», en Ciudad, territorio y paisaje: Reflexiones para un debate multidisciplinar, pp. 63-67.

Martínez, E. (2014): «Configuración urbana, habitar y apropiación del espacio», XIII Coloquio Internacional de Geocrítica El control del espacio y los espacios de control, Universidad de Barcelona, pp. 1-21.

Martínez Taboada, P. (1985): «Desarrollo urbanístico de las ciudades episcopales: Sigüenza en la Edad Media», La España medieval, 7. Universidad Complutense, pp. 957-972.

Montañés, E. (2018): «El furor por los huertos urbanos divide a los científicos», $A B C$ [18/03/2018], s.p.

Monterroso Montero, J. M. (2011): «El Centro Histórico. La creación de una conciencia cultural. El caso de Santiago de Compostela», Actas do Seminário Centros Históricos; Passado e Presente, pp. 39-69.

Moreno Carretero, M. A. (2014): Mecanismo inconscientes del horizonte. Scarpia Ediciones.

Moreno Pérez, J. R..; Márquez Ballesteros, M. J. (2011): «La ciudad contemporánea», Uciencia 8, pp. 38-40.

Navarro, A. (2016): El espacio público como tablero de juego. Conferencia TEDX UP Valencia. https://www.youtube.com/watch?v=bPwWDCbMRTE (consultado el 11 de junio de 2018). 
Navarro, R. (2018 actualizado): «Cinco beneficios reales de tener un huerto urbano», $L a$ Vanguardia [actualizado a 30/05/2018], s.p.

Navarro-Fuentes, C. A. (2016): «La ciudad como espacio de intervención artística», Pensamiento, papeles de filosofía 3, pp. 89-120.

Ortiz Guitart, A. (2007): «Hacia una ciudad no sexista. Algunas reflexiones a partir de la geografía humana feminist para la planeación del espacio urbano», Territorios 16-17, pp. 11-28.

Perniola, M. (2008): Los situacionistas. Historia crítica de la última vanguardia del siglo $X X$. Acuarela \& A. Machado.

Riegl, A. (2017 ed.): El culto moderno de los monumentos. La balsa de la Medusa.

Rodríguez Barcón, A.; Calo García, E.; Otero Enríquez, R. (2017): «Producción cultural y turistificación: la construcción de la ciudad creativa en A Coruña», en Actas Cidades Creativas, Icono 14, pp. 699-710.

Rodríguez Calatayud, N. (2008): «La mujer en los márgenes, la memoria femenina en la ciudad», Diálogos urbanos, confluencias entre arte y ciudad. Universidad de Valencia, pp. 279-288.

Rodríguez Pedret, C. (1991): «Miradas 'pop' en la ciudad contemporánea», Annals 5, pp. 101-111.

Sequera, J. (2015): «A 50 años del nacimiento del concepto 'gentrificación'. La mirada anglosajona», Biblio3W, 1, pp. 1-23.

Sotelo Pérez, M. (2012): «Turismo y ciudades patrimoniales: estudio de caso, Santiago de Compostela», MOA Revista Electrónica de Medioambiente, 12, pp. 62-107.

Sornado, D; Ardura, A. (2016): First we take Manhattan: la destrucción creativa de las ciudades. La Catarata.

Szmulewicz, I. (2017): «La ciudad al margen: más allá del arte público comunitario. Un Proyecto de Ángela Ramírez», Revista Tórax, pp. 1-15.

Verhoeff, N. (2018): «Screens for Urban Data Dramaturgy», Actas $3^{\circ}$ Concreso Internacional Arte, Ciencia y Ciudad, pp. 81-98.

Zukin, S. (1987): «Gentrification: Culture and Capital in the Urban Core», Annual Review of Sociology, 13, pp. 129-147.

Carta de Cracovia. IPCE. http://ipce.mecd.gob.es/dam/jcr:b3b6503d-cf75-4cb0-adaf226740ebd654/2000-carta-cracovia.pdf (consultado el 11 de junio de 2018). UNESCO http://www.unesco.org/culture/natlaws/media/pdf/guatemala/guatemala_carta_cracovia_2000_spa_orof.pdf (consultado el 11 de junio de 2018).

Conferencia de Jaime Lerner en TEDX Buenos Aires (2010). https://www.youtube.com/ watch?v=lXGY0X-wdjI (Consultado el 11 de junio de 2018).

Fundación Huerta de San Antonio https://www.facebook.com/fundacionhuertadesanantonio/ (Consultado el 16 de junio de 2018).

MACBA. Obra de Constant. https://www.macba.cat/es/new-babylon-3207 (Consultado el 11 de junio de 2018). 
Ministerio de Fomento, Gobierno de España. https:/www.fomento.gob.es/MFOM/ LANG_CASTELLANO/DIRECCIONES_GENERALES/ARQ_VIVIENDA/ SUELO_Y_POLITICAS/SOTENIBILIDAD/LBSPUE/ (consultado el 11 de junio de 2018).

ONU, Objetivos de Desarrollo sostenible: Ciudades https://www.un.org/sustainabledevelopment/es/cities/ (Consultado el 11 de junio de 2018).

ONU-Hábitat http://onuhabitat.org.mx/index.php/la-nueva-agenda-urbana-en-espanol (consultado el 11 de junio de 2018). 